\title{
Avaliação do consumo de medicamentos para emagrecer em farmácias, no município de Ceres - Goiás, Brasil
}

Adda Caetana da Silva CRUZ

Edson Negreiros dos SANTOS ${ }^{2}$

1 - Farmacêutica, Especialista em Farmácia Clínica, Farmacêutica das unidades Estratégia da Saúde da Família da

Secretaria Municipal de Saúde de Ceres-Go.

2- Farmacêutico, Mestre em Farmacologia, Coordenador do Curso de Pós Graduação em Farmácia Clínica do Instituto

Pharmacológica. Professor de Farmacologia e Terapêutica na Faculdade de Medicina da PUC- Go.

Autora Responsável: CRUZ, A.C.S

E-mail: addacaetan@hotmail.com

Recebido em: 10/05/2013 - Aprovado em: 30/07/2013 - Disponibilizado em: 15/08/2013

\section{RESUMO}

O presente estudo teve o objetivo de analisar o consumo de medicamentos para emagrecer no município de Ceres, no estado de Goiás. Participaram da pesquisa, clientes de cinco drogarias e de uma farmácia de manipulação. Através destes questionários estudou-se o perfil dos consumidores (gênero, idade, motivo que o levou a fazer uso da medicação), se teve ou não acompanhamento médico, como comprou a medicação e por quanto tempo foi o consumo da mesma. $\mathrm{O}$ resultado do estudo mostrou que em Ceres, as pessoas também procuram emagrecer rapidamente com o objetivo de chegar ao corpo perfeito, não importando se terá qualquer acompanhamento médico.

PALAVRAS-CHAVE: Obesidade. Anorexígenos. Sibutramina. Epidemiologia. Tratamento.

\section{Evaluating consumption of slimming drugs in pharmacies in the city of Ceres - Goiás, Brazil}

\section{ABSTRACT}

The present study aimed to analyze the consumption of slimming drugs in the city of Ceres, in the state of Goiás participated in the research, drugstores and five clients a compounding pharmacy. Through these surveys we studied the profile of consumers (gender, age, reason that led him to take medication), if they had or not medical, bought as medication and how long was the consumption of the same. The result of the study showed that in Ceres, people also seek rapid weight loss in order to reach the perfect body, no matter if have any medical supervision.

KEYWORDS: Obesity. Anorectic. Sibutramine. Epidemiology. Treatment.

\section{INTRODUÇÃO}

A obesidade pode ser definida como o acúmulo excessivo de gordura corporal em extensão tal, que acarreta prejuízos à saúde dos indivíduos, tais como dificuldades respiratórias, problemas dermatológicos e distúrbios do aparelho locomotor, além de favorecer o surgimento de enfermidades potencialmente letais como dislipidemias, doenças cardiovasculares e Diabetes, principalmente a do Tipo II e certos tipos de câncer (MONTEIRO; CONDE, 1999 apud PINHEIRO; FREITAS; CORSO, 2004). É considerada atualmente como um dos mais graves problemas de saúde pública. Sua prevalência vem crescendo acentuadamente nas últimas décadas, inclusive nos países em desenvolvimento, o que levou a doença à condição de epidemia global (CONSENSO LATINO AMERICANO DE OBESIDADE, 1998).

No Brasil, ela atinge $40,6 \%$ da população (IBGE, 2004), e a vontade de possuir um corpo esbelto quer para se adequar aos padrões que a sociedade brasileira exige ou para melhorar a saúde, gera uma procura 
aos consultórios médicos e as farmácias ou drogarias, a fim de encontrar uma saída para o emagrecimento. Mas normalmente, esta perda de peso é procurada e desejada rapidamente, de preferência em dias ou semanas. Desta forma, o uso de anfetaminas se faz, equivocadamente, imprescindível visando atender esta "procura desenfreada".

O Consenso Latino Americano de Obesidade considera esta uma enfermidade crônica e que quando "mudanças no estilo de vida" não produzem os efeitos desejados e/ou co-morbidades podem colocar em risco a vida do paciente é necessário a intervenção farmacoterapêutica. Assim, nestes casos considera-se necessário o uso de medicamentos como tratamento coadjuvante, mantendo-se ainda o foco na "mudança de estilo de vida”. Para uma adequada prescrição de medicamentos, deve ser consideradas pacientes com:

- Índice Massa Corpórea: igual ou maior a 30 ;

- Índice Massa Corpórea: igual ou maior que 25, mas acompanhado de fatores de risco como a Hipertensão Arterial, Diabetes Mellitus tipo 2, Hiperlipidemia, etc;

-Quando não alcançados os objetivos somente com planos alimentares e estímulo a atividades físicas, ou seja, que o paciente não tenha perdido $1 \%$ do peso inicial por mês, após um a três meses de tratamento não medicamentoso.
As premissas fundamentais para a indicação da farmacoterapia no tratamento da obesidade são:

- A medicação não deve constituir um critério único de tratamento;

- Deve estar enfocada no tratamento integral do paciente obeso e não exclusivamente na redução de peso;

- O tratamento deve ser prescrito e acompanhado por um médico.

Segundo, a I Diretriz Brasileira de Diagnóstico e Tratamento da Síndrome Metabólica, em 2007, haviam cinco medicamentos registrados no Brasil para o tratamento da obesidade: anfepramona (dietilpropiona), femproporex, mazindol, sibutramina e orlistat.

A sibutramina foi testada em vários estudos. Um estudo bem controlado, com duração de dois anos, demonstrou que este medicamento é eficaz na perda de peso com melhora dos parâmetros metabólicos, boa tolerabilidade e segurança. A dose preconizada varia de $10 \mathrm{mg}$ a $20 \mathrm{mg}$ por dia (JAMES et al., 2000). Efeitos colaterais: boca seca, constipação intestinal, insônia, irritabilidade e cefaléia. Aumentos médios de pressão arterial sistólica e diastólica e de freqüência cardíaca também têm sido relatados. Recomenda-se controle rigoroso da pressão arterial e da freqüência cardíaca e ajuste da medicação anti-hipertensiva, quando necessário (ARTERBURN; CRANE; VEENSTRAI, 2004). 
Os estudos com anfepramona e mazindol demonstram que estes medicamentos são eficazes na perda de peso, entretanto, estes estudos são na maioria antigos (30 ou mais anos) e de curto prazo. Atualmente, são encontrados poucos estudos clínicos publicados com o femproporex. As doses recomendadas são: anfepramona $50 \mathrm{mg}$ a 100mg, femproporex $25 \mathrm{mg}$ a $50 \mathrm{mg}$ e mazindol $1 \mathrm{mg}$ a $3 \mathrm{mg}$. Tendo como efeitos colaterais: sintomas leves a moderados de estimulação do sistema nervoso central (insônia, nervosismo, euforia) e do sistema cardiovascular (taquicardia e, ocasionalmente, elevação da pressão arterial). Assim, devido à ausência de estudos de longa duração (mais de um ano), não há evidência da eficácia e segurança do uso destes medicamentos em longo prazo (I Diretriz Brasileira de Diagnóstico e Tratamento da Síndrome Metabólica).

Ainda segundo, a I Diretriz Brasileira de Diagnóstico e Tratamento da Síndrome Metabólica, não são encontrados estudos sobre a associação de drogas, entretanto, por não ter ação central, o orlistat poderia ser associado a qualquer um dos outros medicamentos. Mas, não se deve associar os catecolaminérgicos entre si ou com a sibutramina.

Já os inibidores seletivos de
recaptação de serotonina, mesmo sendo
usados para o tratamento de depressão,
(fluoxetina, sertralina) podem proporcionar o

efeito de perda de peso, embora não estejam aprovados para o tratamento da obesidade. A fluoxetina demonstrou um efeito transitório de perda de peso, presente principalmente nos seis primeiros meses de uso, após o qual pode ocorrer recuperação do peso perdido. A dose empregada de fluoxetina é de $20 \mathrm{mg}$ a $60 \mathrm{mg}$ e a de sertralina, 50mg a $150 \mathrm{mg}$ por dia. Efeitos colaterais: ansiedade, insônia, sonolência, diminuição da libido, tremores e alteração da memória (DARGA et al, 1991).

\section{METODODOLOGIA}

$\mathrm{O}$ presente estudo foi realizado em cinco drogarias e em uma farmácia de manipulação, no município de Ceres, Goiás, no período de novembro a dezembro de 2007. Como instrumento de coleta de dados, foi usado um questionário estruturado preenchido por cliente de cada farmácia envolvida no projeto. A cada empresa foi entregue um envelope com 35 questionários com 1 caneta, com a seguinte explicação:

- Qualquer pessoa que entre na empresa poderá preencher o questionário, independente se já usou ou não medicamento para emagrecer.

- Informar ao cliente que este questionário será usado como base para trabalho de conclusão de curso de Especialização em Farmácia Clínica, podendo ser preenchido pelo cliente de forma anônima e espontânea e colocado em uma urna. 


\section{RESULTADOS E DISCUSSÃO}

104 questionários foram respondidos, dos 210 questionários entregues. Sendo que 60 clientes afirmaram ter consumido algum medicamento para emagrecer. Os clientes do sexo feminino destacaram-se como os grandes usuários deste tipo de medicamento, atingindo $78,68 \%$ dos pesquisados. Esta situação pode ocorrer, provavelmente em função de vários fatores, entre eles o fato de as mulheres serem muito influenciáveis pela mídia e pela sociedade, que cultuam corpos extremamente magros. Este fato promove uma busca incessante do corpo perfeito em pouco tempo e sem sofrimento (CARAZZATTO, 2003).

De acordo com a Figura 1, observa-se que grande parte dos consumidores de medicamentos para emagrecer tem faixa etária de 31 a 40 anos, atingindo 42,62\% dos pesquisados, seguida da faixa etária de 21 a 30 anos, $34,43 \%$.

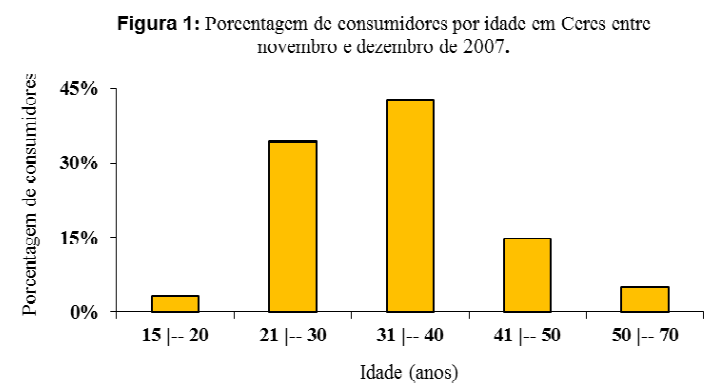

Ao observarmos a Figura 2, nota-se que $42,62 \%$ dos clientes pesquisados relataram que usaram o medicamento para reduzir o sobrepeso, atingindo os padrões estéticos da sociedade e melhorando a autoestima. Enquanto que 36,07\% usaram o medicamento para reduzir o sobrepeso a fim de melhorar a saúde. A mídia estimula o padrão estético magro, discriminando o gordo de uma maneira não sutil, com mensagens agressivas, persuasivas e pouco estimuladoras, reforçando a baixa autoestima nos indivíduos obesos. Evidencia-se, ainda, que em jornais e revistas aparecem em número mais significativo mensagens e textos de conotação negativa e discriminatória do que aspectos que tratem da obesidade como doença, definindo-a como um problema de saúde (FELIPPE et al., 2004).

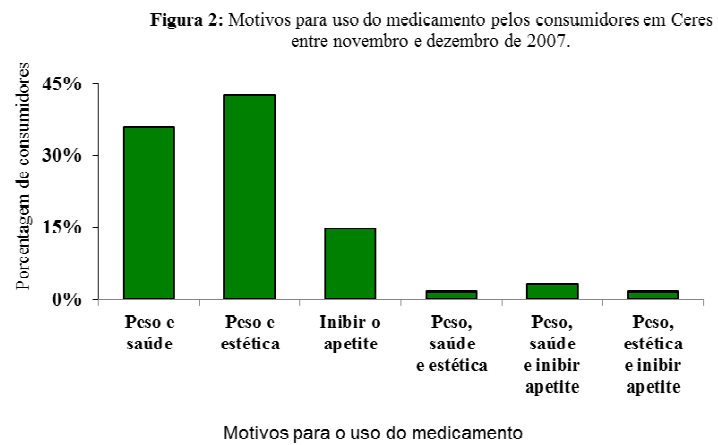

No levantamento realizado verificouse que $42,62 \%$ dos pesquisados relataram que utilizaram o femproporex (26 pacientes), outros 22,95\% (14 pacientes) usaram sibutramina e $13,11 \%$ utilizaram anfepramona (8 pacientes) (Figura 3). Também $6,56 \%$ descreveram que utilizaram associação de femproporex com anfepramona (4 pacientes), e $4,92 \%$ femproporex com sibutramina (3 pacientes), mesmo sendo proibido a prescrição e o aviamento de fórmulas contendo associação medicamentosa das 
substâncias anorexígenas entre si ou com ansiolíticos, diuréticos, hormônios ou extratos hormonais e laxantes, bem como quaisquer outras substâncias com ação medicamentosa, segundo a Portaria 344/98, no seu art. 47.

Os medicamentos anorexígenos de ação central devem ser utilizados por curto prazo, tanto por seus efeitos colaterais como por seu elevado potencial para abuso e dependência, alem de desencadear sintomas e síndromes psiquiátricas, como surtos psicóticos, síndromes depressivas ou de mania (VASQUES; MARTINS e DE AZEVEDO, 2004). Estudos têm mostrado segurança do uso de sibutramina por períodos de até 18 meses consecutivos, pois mesmo a sibutramina provocando efeitos colaterais, estes são mais brandos e de menor duração que os associados aos medicamentos noradrenérgicos (NONINO-BORGES; BORGES e DOS SANTOS, 2006).
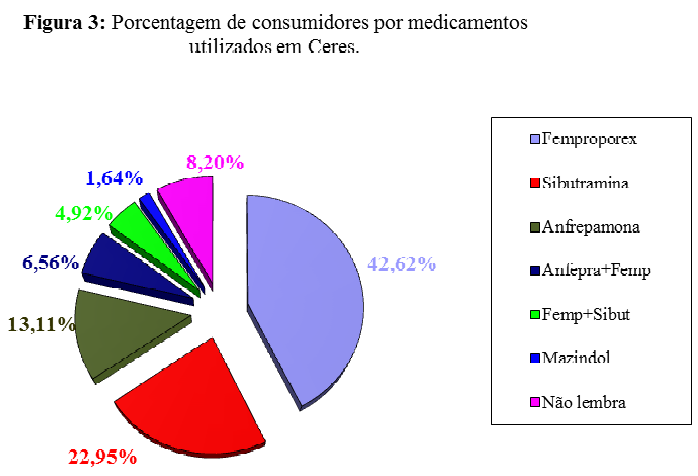

Ainda neste levantamento, observouse que apenas $16,40 \%$ dos pesquisados teve acompanhamento de um médico endocrinologista por todo tratamento, $37,70 \%$ declaram que não tiveram acompanhamento médico e $45,9 \%$ dos pesquisados foram acompanhados por profissionais de outra especialidade médica, como: ginecologia, dermatologia, otorrinolaringologia, clinico geral, nutrologia, urologia, cardiologia e neurologia.

Os pesquisados ainda informaram como compraram o medicamento para emagrecer. Cerca de 59\% utilizaram receituário especial entregue pelo médico, $20 \%$ adquiriram o medicamento sem receituário, $13 \%$ pagaram pela consulta apenas para conseguir a receita especial e 5\% com receituário de outra pessoa.

\section{CONCLUSÃO}

Diante do exposto sobre os medicamentos usados no emagrecimento, e após pesquisa do perfil dos consumidores, no município de Ceres, de tais drogas, ficou evidente que:

- O medicamento mais consumido foi o femproporex seguido pela sibutramina;

- Grande parte dos consumidores foram mulheres adultas, cujo objetivo com o consumo era de perder peso para chegar aos padrões que a sociedade impõe;

- A maioria usou o receituário especial para comprar a medicação, mas poucos tiveram um acompanhamento com um médico endocrinologista;

Estes dados de 2007 fundamentam a preocupação da Agência Nacional de 
Vigilância Sanitária com a prescrição, o aviamento ou a dispensação de medicamentos ou fórmulas medicamentosas que contenham substâncias psicotrópicas anorexígenas e para isso lança mão de várias portarias e Resoluções RDC (Resolução da Diretoria Colegiada), a fim de controlar melhor estes medicamentos especiais, como a RDC $\mathrm{N}^{\circ} 52$, de 6 de outubro de 2011, proibindo o uso das substancias anfepramona, femproporex e mazindol, bem como seus intermediários e aumentando o controle da prescrição e dispensação de medicamentos que contenham a sibutramina.

\section{REFERÊNCIAS}

AGÊNCIA NACIONAL DE VIGILÂNCIA SANITÁRIA. Ministério da Saúde (Brasil).

PORTARIA 344 DE 12 DE MAIO DE 1998. Aprova o Regulamento Técnico sobre substâncias e medicamentos sujeitos a controle especial. Disponível em: <http://www.anvisa.gov.br/e-legis/>. Acesso em: 20 abr 2007.

AGÊNCIA NACIONAL DE VIGILÂNICA SANITÁRIA. Ministério da Saúde (Brasil). Sistema Nacional para Gerenciamento de Produtos Controlados. Brasília-DF: ANVISA, 2007.

Disponível em: <http://www.anvisa.gov.br/hotsite/sngpc/historico.htm>. Acesso em: 24 ago. 2007.

ARTERBURM, D. E.; CRANE, P. K.; VEENSTRA, D.L. The efficacy and safety of sibutramine for weight loss: a systematic review. Archive International Medicinal, v. 164, p. 994-1003, 2004.

ASSOCIAÇÃO BRASILEIRA DE NORMAS TÉCNICAS (Brasil). Informação e documentação

- Referências - Elaboração (NBR 6023). Rio de Janeiro, 2002. 21p.

ASSOCIAÇÃO BRASILEIRA DE NORMAS TÉCNICAS (Brasil). Informação e documentação - Citações em documentos - Apresentação (NBR 10520). Rio de Janeiro, 2002. 5p.

ASSOCIAÇÃO BRASILEIRA DE NORMAS TÉCNICAS (Brasil). Informação e documentação - Trabalhos acadêmicos - Apresentação (NBR 14724). Rio de Janeiro, 2002. 21p.

BRASIL, Resolução RDC n. 52, de 06 de outubro de 20011. Dispõe sobre a proibição do uso das substâncias anfepramona, femproporex e mazindol, seus sais e isômeros, bem como intermediários e medidas de controle da prescrição e dispensação de medicamentos que contenham a substância 
sibutramina, seus sais e isômeros, bem como intermediários e dá outras providências. D.O.U. -

Diário Oficial da União; Poder Executivo. Brasil 10 de outubro de 2011.

CARAZZATTO, P. R. A Farmácia Magistral e o Tratamento Farmacoterápico da Obesidade.

Racine, São Paulo, n. 77, p. 34-40, 2003.

CONSENSO LATINO AMERICANO DE OBESIDADE, Rio de Janeiro, Brasil, 1998.

Disponível em: <http://www.abeso.org.br/pdf/consenso.pdf>. Acesso em: 9 abr. 2007.

Darga L. L.; Carroll-Michals, L.; Botsford, S. J; LUCAS, C. P. Fluoxetine's effect on weight loss in obese subjects. American Journal of Clinical Nutrition, XXX, v. 54, p. 321-325, 1991.

FELIPPE, F. M. L; FRIEDMAN, R.; ALVES, B. S.; CIBEIRA, G. H.; SURITA, L. E.; TESCHE, C. Obesidade e Mídia: o lado sutil da informação. Revista Acadêmica do Grupo Comunicacional de São Bernardo, São Paulo, n. 2, 2004. Disponível em:

<http://www.metodista.br/unesco/GCSB/artigo_obesidade_midia.pdf/>. Acesso em: 16 abr. 2007.

INSTITUTO BRASILEIRO DE GEOGRAFIA E ESTATÍSTICA (Brasil). Senso populacional estimado em 2006. Brasília-DF. IBGE, 2006. Disponível em

<www.ibge.gov.br/cidadesat/topwindow.htm?/>. Acesso em 24 ago. 2007.

INSTITUTO BRASILEIRO DE GEOGRAFIA E ESTATÍSTICA (Brasil). Obesidade atinge mais de $40 \%$ da população brasileira. Brasília-DF. IBGE, 2004. Disponível em <www.ibge.gov.br/ibgeteen/noticias/obesid.html>. Acesso em 24 ago. 2007.

James, W. P.; Astrup, A.; Finer, N.; Hilsted, J.; Kopelman, P.; Rössner, S.; Saris, W. H.; Van Gaal, L. F. Effect of sibutramine on weight maintenance after weight loss: a randomized trial. The Lancet, XXXXX, v. 356, p. 2119-2125, 2000

JIFE - Relatório da Junta Internacional de Fiscalização de Entorpecentes (JIFE), lançado em parceria com o Escritório das Nações Unidas contra Drogas e Crime (UNODC), Rio de Janeiro, 1 mar, 2007. Disponível em: < http://www.unodc.org/pdf/brazil/5-

Rem\%E9dios\%20Incentivam\%20Obssess\%E3o\%20Pela\%20Magreza,\%20Alerta\%20a\%20Junta.d oc>. Acesso em: 29 jan. 2008 
-BORGES C. B.; BORGES R. M.; DOS SANTOS J. E. Tratamento Clínico da Obesidade.

Medicina, Ribeirão Preto, v. 2, p. 246-252, 2006. Disponível em: <

http://www.fmrp.usp.br/revista/2006/vol39n2/10_tratamento_clinico_obesidade1.pdf> Acesso em: 19. abr. 2007.

PINHEIRO, A. R. O.; FREITAS, S.; CORSO, A. C. T. Uma abordagem epidemiológica da obesidade. Revista de Nutrição, Campinas, v. 17, n. 4, p. 523-33, 2004. Disponível em: < http://www.scielo.br/pdf/rn/v17n4/22900.pdf>. Acesso em: 19 abr. 2007.

SALOMONE, R. Um show de descontrole. Revista Veja, São Paulo,1946 ed. 2006. Disponível em <http://veja.abril.com.br/080306/p_078.html>. Acesso em 27 ago. 2007.

SILVA, P. Farmacologia. In: Augello, D. E. Estimulante do sistema Nervoso Central. Hiperatividade. Narcolepsia. Obesidade. Estimulantes Respiratórios. Rio Janeiro: Guanabara Koogan, 2002. cap. 45, p. 422-430.

SOCIEDADE BRASILEIRA DE CARDIOLOGIA (Brasil). I Diretriz Brasileira de Diagnóstico e Tratamento da Síndrome Metabólica. Arquivos Brasileiros de Cardiologia, São Paulo, v. 84, 2005. Disponível em: <http://publicacoes.cardiol.br/consenso/2005/sindromemetabolica.pdf>. Acesso em 21 abr. 2007.

SOCIEDADE BRASILEIRA DE ENDOCRINOLOGIA E METABOLOGIA. Obesidade:

Tratamento. 17 abr. 2006. Disponível em: < http://www.projetodiretrizes.org.br/4_volume/23ObesidadeTratamento.pdf>. Acesso em: 20 abr. 2007.

VASQUES, F.; MARTINS, F.C.; DE AZEVEDO, A.P. Aspectos psiquiátricos do tratamento da obesidade. Revista de Psiquiatria Clinica, São Paulo, v. 4, p. 195-98, 2004. disponível em: < http://www.scielo.br/pdf/rpc/v31n4/22408.pdf>. Acesso em: 19 abr. 2007. 